\title{
Rapid eye-fixation training without eyetracking
}

\author{
Emmanuel GuZman-Martinez \\ Northwestern University, Evanston, Illinois \\ and Universidad Nacional Autonóma de México, Mexico City, Mexico \\ AND \\ Parkson Leung, Steve Franconeri, Marcia Grabowecky, and Satoru Suzuki \\ Northwestern University, Evanston, Illinois
}

\begin{abstract}
Maintenance of stable central eye fixation is crucial for a variety of behavioral, electrophysiological, and neuroimaging experiments. Naive observers in these experiments are not typically accustomed to fixating, either requiring the use of cumbersome and costly eyetracking or producing confounds in results. We devised a flicker display that produced an easily detectable visual phenomenon whenever the eyes moved. A few minutes of training using this display dramatically improved the accuracy of eye fixation while observers performed a demanding spatial attention cuing task. The same amount of training using control displays did not produce significant fixation improvements, and some observers consistently made eye movements to the peripheral attention cue, contaminating the cuing effect. Our results indicate that (1) eye fixation can be rapidly improved in naive observers by providing real-time feedback about eye movements, and (2) our simple flicker technique provides an easy and effective method for providing this feedback.
\end{abstract}

In many visual experiments, maintenance of central eye fixation is crucial. Some experiments require that stimuli be presented at specific locations on the retina (e.g., to investigate hemispheric or eccentricity-based differences in visual processing). Other experiments aim to manipulate only covert visual attention and need to minimize overt eye movements. Eye movements also cause artifacts in EEG recordings and can lead to loss of trials. Eye fixation can be improved by monitoring eye movements with an eyetracker and providing feedback when eye movements occur (e.g., Steinman, Haddad, Skavenski, \& Wyman, 1973). Our goal was to devise an easy and effective method that does not require the use of an eyetracker and that rapidly trains naive observers to maintain central eye fixation, even under challenging conditions.

We took advantage of an eye-movement-contingent visual effect. Individual flashes of a rapidly flickering dot, which are too fast to resolve when the eyes are stationary, are seen as a spatially displaced array of flashes during the fast flight of a saccadic eye movement, since each flash is painted at a different location on the retina (e.g., Hershberger, 1987). We adapted this phenomenon into a display that generated a clear visual effect whenever observers made even a small eye movement (e.g., a saccade, a mechanically induced [e.g., by tapping on the head or the side of the eye] movement of the eye, a blink).

Our display consisted of a fine-grained random dot pattern (with $50 \%$ black and $50 \%$ white pixels) that flick- ered rapidly $(37.5 \mathrm{~Hz}$, the fastest rate possible using a typical $75-\mathrm{Hz}$ monitor) in counterphase (i.e., all black pixels became white and all white pixels became black in alternate frames) (see Figure 1). When the eyes were stationary, the display appeared uniformly gray, because the black and white pixels were perceptually averaged at each location (except for some graininess perceived because of the visual system's sensitivity to borders defined by regions flickering in opposite phases; e.g., Forte, Hogben, \& Ross, 1999).

As soon as the observer made even a small eye movement, the precise temporal averaging of the black and white pixels was disrupted, because individual frames fell at shifted locations on the retina, and the observer saw a clear black-and-white random dot pattern momentarily popping out from the gray field. Because naive observers are often unaware of their eye movements and blinks, we thought that this eye-movement-contingent effect might provide a useful form of feedback for training naive observers to maintain steady central eye fixation by making them aware of their unintended eye movements in real time.

We determined whether a few minutes of training with this flicker display substantially improved central eye fixation in naive observers. We measured the effect of this training using a typical spatial attention cuing task (see Funes, Lupiáñez, \& Milliken, 2005, for a review), in which a peripheral onset cue was flashed to the left or right of a central fixation marker, and the subsequent target stimulus was presented on the cued or opposite side

S.Suzuki, satoru@northwestern.edu 


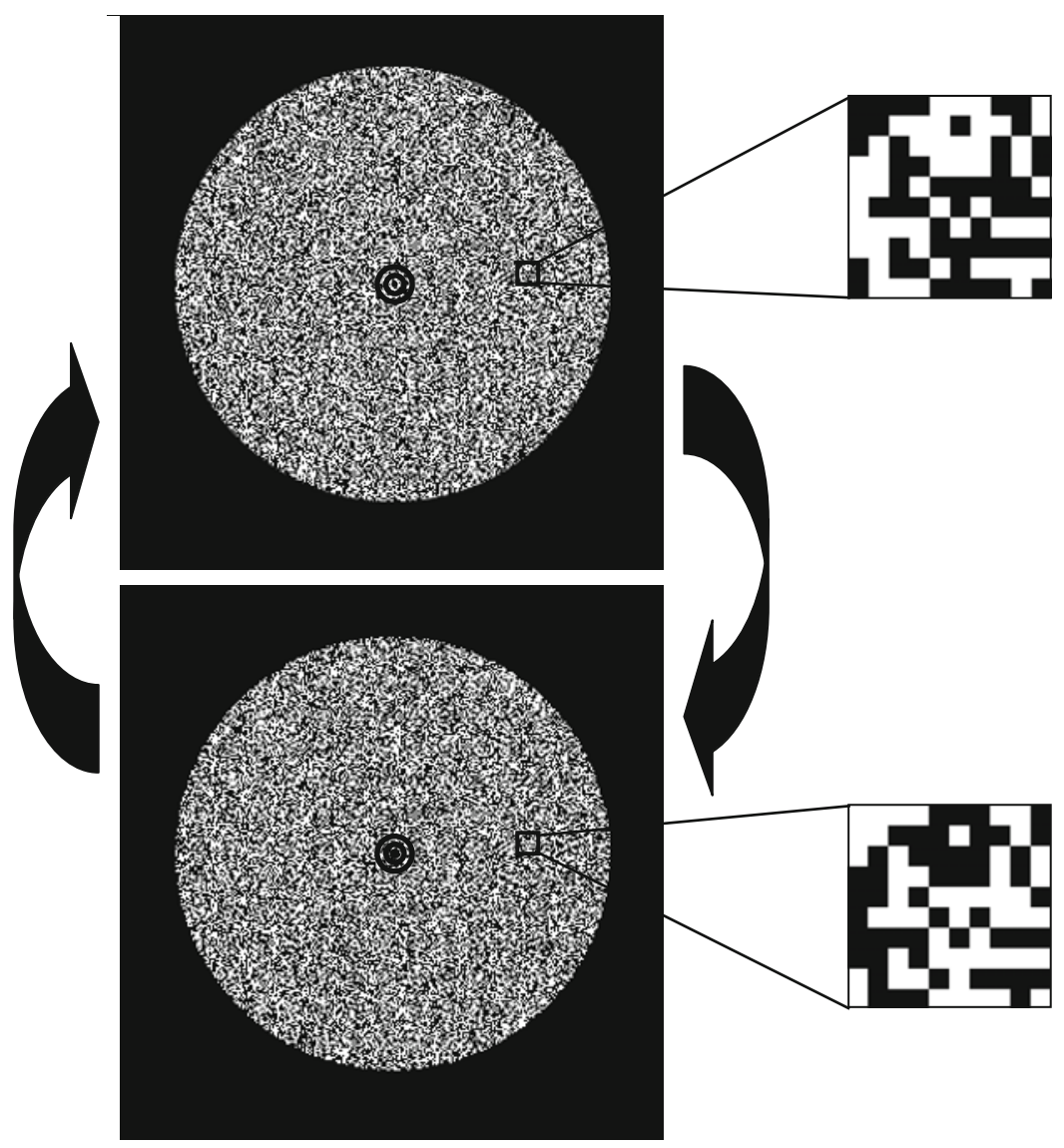

Figure 1. An illustration of the flicker pattern used for fixation training. A random dot pattern (with $50 \%$ black pixels and $50 \%$ white pixels, each pixel subtending $0.047^{\circ}$ visual angle) was rapidly $(37.5 \mathrm{~Hz}, 13.3 \mathrm{msec} /$ frame) alternated with its contrast-reversed complement (i.e., the black pixels became white and the white pixels black; see the magnified illustrations on the right). When the eyes were stationary, the display appeared uniformly gray. However, a random dot pattern momentarily popped out whenever the observer made an eye movement, providing real-time visual feedback on involuntary eye movements (see the text for details).

(Figure 2). Observers responded as to whether the target (an arrow shape) was pointing up or down and were instructed to maintain central eye fixation during a fairly long $(3,200-\mathrm{msec})$ period from the start of the trial (initiated by the observer's buttonpress) to the offset of the target stimulus. The abrupt onset of the peripheral attention cue, the temptation to look at the cued location because of the high ( $80 \%)$ cue validity, and the long (1,500-msec) stimulus onset asynchrony (SOA) between the attention cue and the target stimulus all made it challenging for naive observers to maintain central eye fixation.

During the fixation-training period (lasting a few minutes), observers in the experimental group were informed that whenever they saw a jagged random dot pattern briefly popping out of the gray field, they had made an eye movement, and they were asked to try to reduce eye movements as much as possible (the informed-flicker training condition). We also ran two separate control groups of observers. Observers in one control group were asked to practice fixating the central fixation marker while looking at the flicker display (as in the informedflicker training condition), but they were not informed of the fact that the popping out of random dot patterns was a consequence of their eye movements; they were instead told that the display would occasionally be disrupted because of a computer problem (the misinformed-flicker control condition). Observers in the second control group saw only the central fixation marker during training (in the absence of the flicker display) and were asked to practice fixating the fixation marker (the no-flicker control condition).

Our goal was to demonstrate that naive observers could rapidly and substantially improve central eye fixation when they were given eye-movement-contingent visual feedback during the informed-flicker training. The misinformed-flicker condition controlled for the potential effect of being exposed to the flicker stimulus while trying to improve fixation; the no-flicker condition controlled for the effect of simply trying to improve fixation during the training period. 


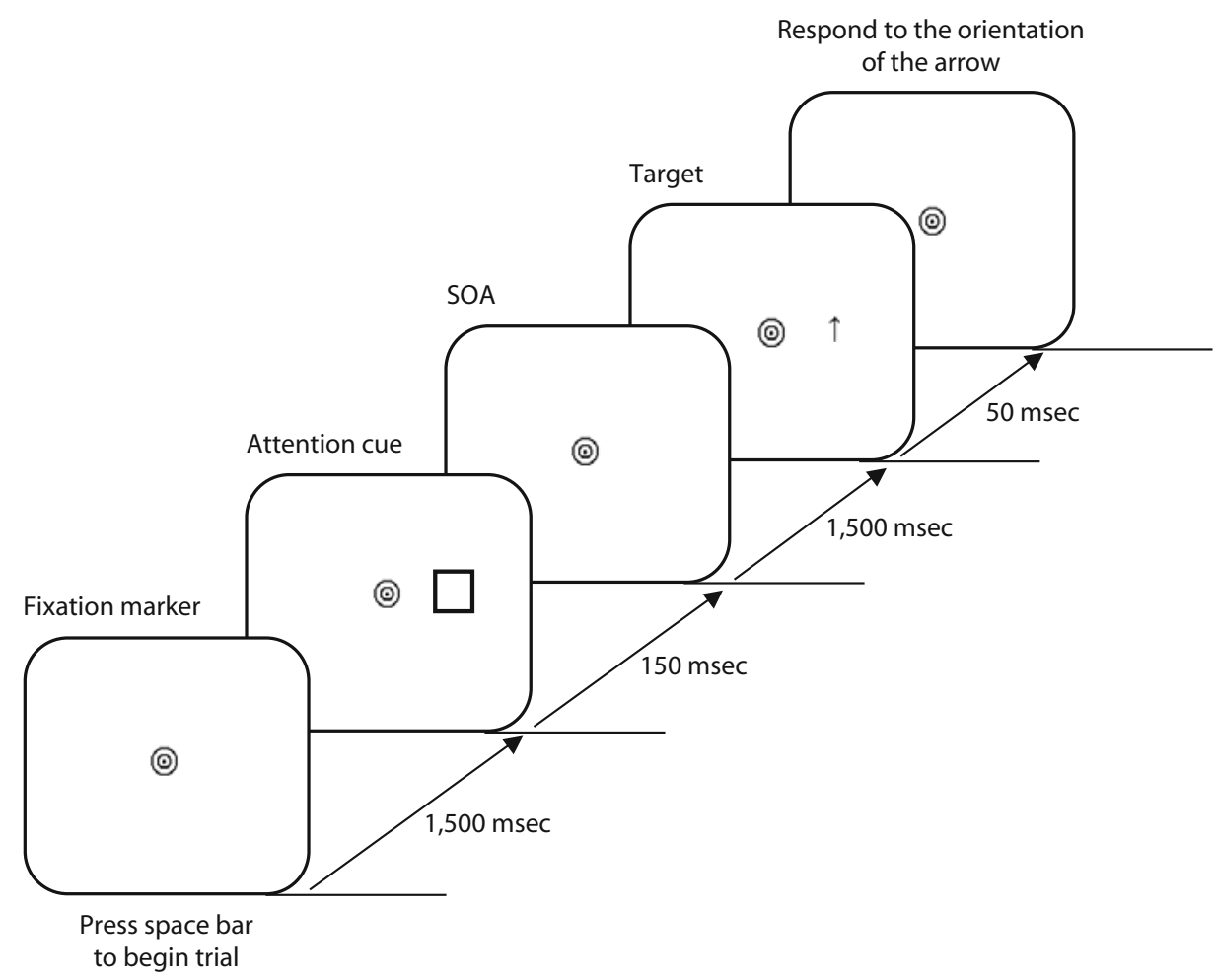

Figure 2. An illustration of a trial sequence for the attention task. The attention cue was flashed to the left or right of the fixation marker with equal probability. The target (an up or down arrow) was presented at the cued location on $80 \%$ of the trials; on the remaining $20 \%$ of the trials, the target was presented on the opposite side of the fixation marker. Observers were instructed to maintain central eye fixation from the beginning of the trial through the offset of the target stimulus; eye movements were monitored throughout this $3,200-$ msec period. SOA, stimulus onset asynchrony.

\section{METHOD}

\section{Observers}

Thirty-six Northwestern University students gave informed consent to participate for partial course credit or monetary compensation. Observers were randomly assigned to the informed-flicker training or to one of the two control conditions (misinformed-flicker or no-flicker control), with the following constraint. Observers were assigned to these conditions on the basis of their fixation performance in the initial attention task, so that the average level of fixation performance before training was similar across the three conditions (see Figure 3).

\section{Stimuli and Procedure}

All stimuli were presented on a 19-in. CRT monitor driven at $75 \mathrm{~Hz}$, at a viewing distance of $42 \mathrm{~cm}$. The bull's-eye fixation marker was $1.27^{\circ}$ in diameter (the diameters of the inner circles were $0.69^{\circ}$ and $0.21^{\circ}$ ).

The attention task. The stimuli were drawn in black against a white background. The attention cue was a $2.87^{\circ} \times 2.87^{\circ}$ square presented $9.76^{\circ}$ to the left or right of the fixation marker. The target arrow $\left(1.27^{\circ}\right.$ in length and $0.55^{\circ}$ in width, pointing up or down) was presented either at the cued location ( $80 \%$ of the time) or at the opposite location ( $20 \%$ of the time). The two cue locations (left and right) and two directions of the target arrow (up and down) were equally probable and randomly intermixed across the 40 trials. Details of the timing of trial events are shown in Figure 2. Observers were instructed to report whether the target arrow was pointing up or down as quickly and accurately as possible by pressing the corresponding keys on the computer keyboard. They were also in- structed to maintain central eye fixation from the beginning of the trial through the offset of the target stimulus. Eye movements were recorded throughout this 3,200-msec trial period with an EyeLink 1000 eyetracker (SR Research, $0.25^{\circ}$ resolution).

For each observer, we computed the attention cuing effect by subtracting the mean response time (RT) for the cue-valid trials from the mean RT for the cue-invalid trials (a higher positive value indicating a stronger cuing effect). The errors were very small and slightly higher in the cue-invalid condition $(0.018$ in the cue-valid condition and 0.028 in the cue-invalid condition), providing no evidence of a speed-accuracy trade-off. The RT outliers were eliminated on the basis of a $3 S D$ criterion for each condition (cue valid and cue invalid) for each observer, but the trimming did not affect the overall statistical results.

Fixation training and control tasks. The entire fixation training lasted $150 \mathrm{sec}$, and it was given in 30 intervals $(5 \mathrm{sec}$ per interval), so that observers had ample opportunity to take breaks. Each training interval was initiated by the observer's buttonpress. In the informed-flicker training condition, observers viewed a flickered random dot pattern, with $50 \%$ of the pixels black and $50 \%$ white; each pixel subtended $0.047^{\circ}$. The random dot pattern was presented in a circle $\left(17.27^{\circ}\right.$ in diameter) with a black bull's-eye fixation marker in the center and was rapidly flickered at $37.5 \mathrm{~Hz}$ in counterphase (i.e., all black pixels became white and all white pixels became black in alternate frames) (Figure 1). The flickered random dot pattern appeared gray when the eyes were stationary because of temporal summation of black and white pixels, but this summation was disrupted when observers moved their eyes, resulting in momentary perception of a jagged random dot pattern. Observers were informed that, every time they saw a random dot pattern pop 


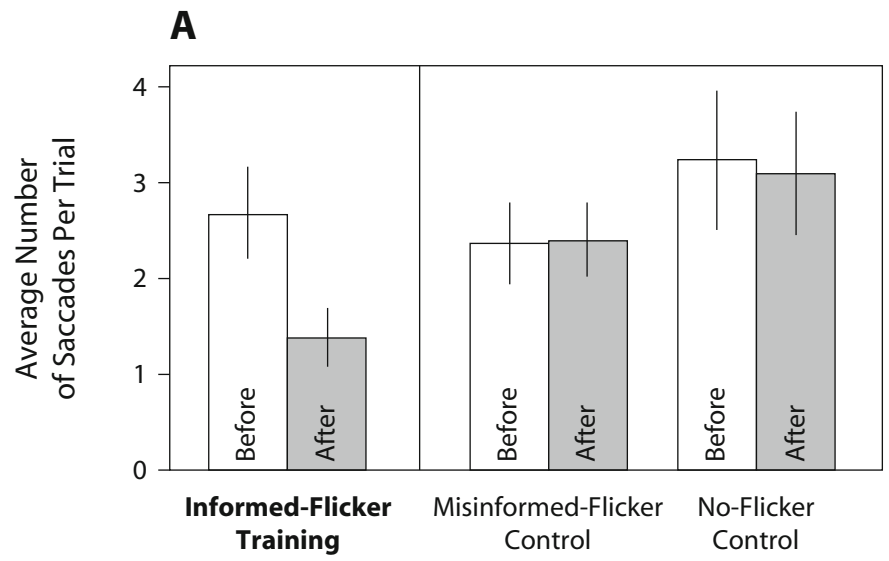

B

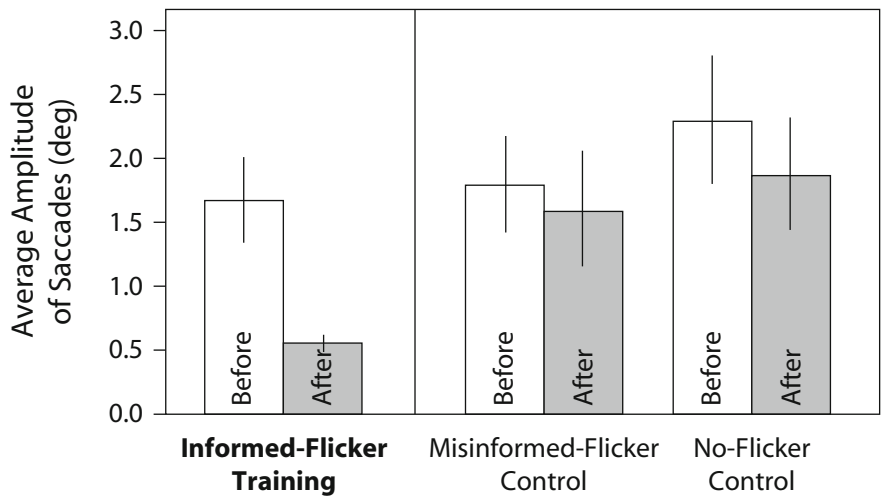

Figure 3. Measures of eye movements during the attention task. (A) The average number of saccades per trial before (white bars) and after (gray bars) undergoing the informed-flicker training (left), the misinformedflicker control (middle), or the no-flicker control (right). (B) The average amplitude of saccades (in degrees of visual angle) before (white bars) and after (gray bars) undergoing the informed-flicker training (left), the misinformed-flicker control (middle), or the no-flicker control (right). Note that only the informed-flicker training improved fixation performance. The error bars represent one standard error of the mean.

out from the gray field, this indicated that they had made an eye movement, and they were instructed to use this feedback to minimize eye movements.

In the misinformed-flicker control condition, observers viewed the identical display. However, these observers were told that the random dot patterns that popped out were generated by an error in the computer program and were instructed to ignore them and to practice maintaining eye fixation on the central bull's-eye marker. In the no-flicker control condition, observers viewed a white central bull's-eye fixation marker presented against a black background and were instructed to practice maintaining eye fixation on the bull's-eye marker. Data for the attention experiment were collected both before and after the fixation training or one of the control conditions.

\section{RESULTS}

Central eye fixation substantially improved following the informed-flicker training. The average number of saccades (made during the 3,200-msec period from the beginning of the trial through the offset of the target stimulus) decreased from 2.63 to $1.35[t(11)=5.13, p<$ $.0005, d=1.48]$ (the left graph in Figure 3A). The average amplitude of saccades was also substantially reduced from $1.66^{\circ}$ to $0.55^{\circ}[t(11)=3.65, p<.005, d=1.05]$ (the left graph in Figure 3B). No improvement occurred following the misinformed-flicker control; the average number of saccades changed from 2.32 to $2.36[t(11)=$ 0.23 , n.s., $d=0.06$ ] (the middle graph in Figure $3 \mathrm{~A}$ ), and the average amplitude of saccades changed from $1.78^{\circ}$ to $1.58^{\circ}[t(11)=1.01$, n.s., $d=0.29]$ (the middle graph in Figure $3 \mathrm{~B}$ ). None occurred following the no-flicker control either; the average number of saccades changed from 3.21 to $3.08[t(11)=0.41$, n.s., $d=0.12]$ (the right graph in Figure $3 \mathrm{~A}$ ), and the average amplitude of saccades changed from $2.29^{\circ}$ to $1.85^{\circ}[t(11)=1.38$, n.s., $d=0.40]$ (the right graph in Figure 3B). Thus, the informed-flicker training substantially improved central eye fixation by significantly reducing both the number and amplitude of saccades. In contrast, simply asking observers to practice 

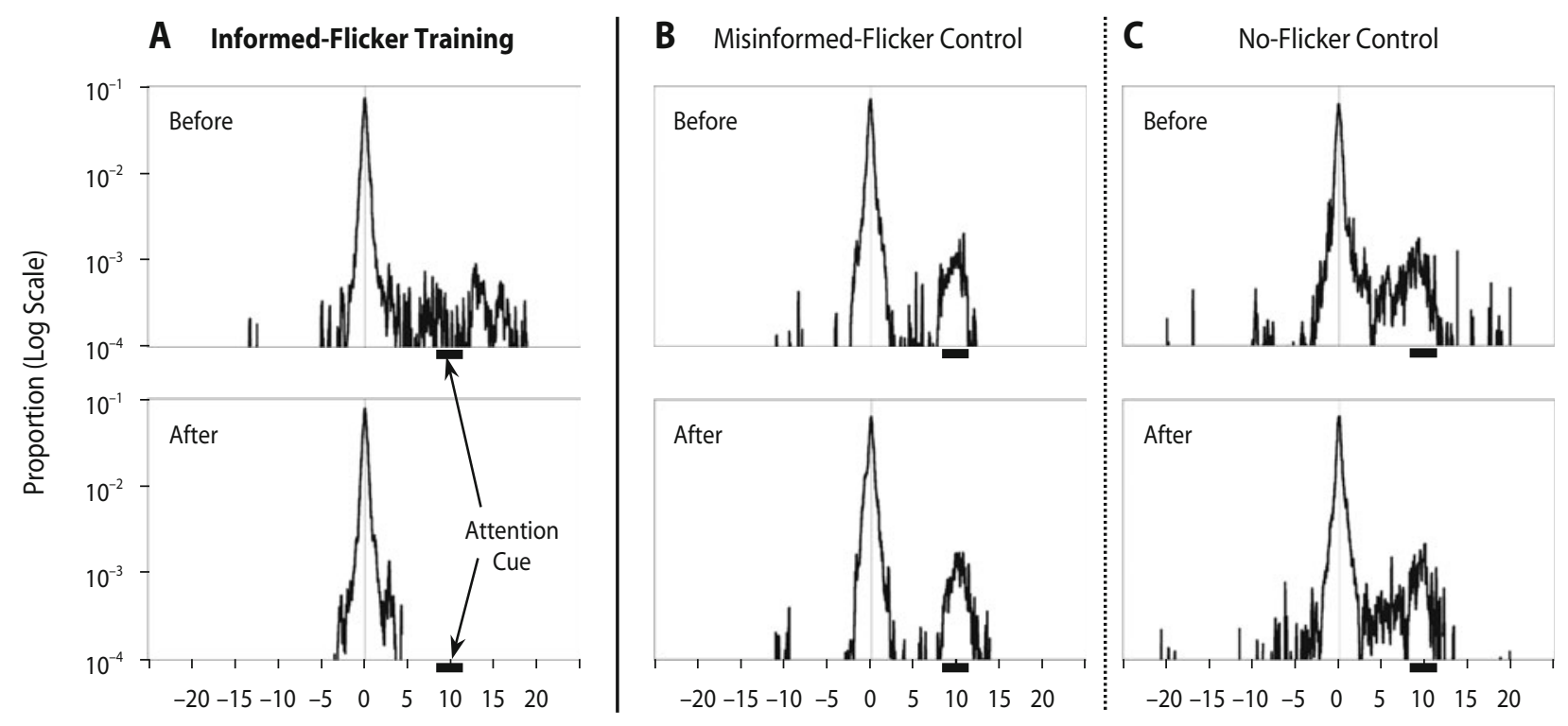

Horizontal Eye Position During Trial (deg)

Figure 4. Histograms of horizontal eye position before (upper panels) and after (lower panels) the informed-flicker training (A), the misinformed-flicker control (B), or the no-flicker control (C). The proportions (the $y$-axis) are shown in log scale to highlight eye movements toward the attention cue, which were relatively infrequent. The horizontal bar along the $x$-axis represents the attention cue. The data shown have been averaged across observers and the cued location (left and right). Note that observers made eye movements toward the attention cue during intended fixation (upper panels) and that these eye movements toward the cue were eliminated after the informed-flicker training (the lower panel in A).

central fixation with or without the flicker display had little effect.

Histograms of horizontal eye position (sampled every $8 \mathrm{msec}$ on each trial, and averaged across observers and the left and right cue locations) show that eye movements prior to fixation training were systematically directed toward the attention cue (the upper row in Figure 4). Note that the proportions ( $y$-axis) are shown in log scale to highlight eye movements, because observers successfully fixated during large portions of each trial. The patterns of eye movements showing a secondary peak around the cued location remained essentially the same before and after the misinformed-flicker control (Figure 4B) and the no-flicker control (Figure 4C), indicating that the control conditions did not reduce eye movements toward the attention cue. In contrast, the informed-flicker training virtually eliminated eye movements toward the cue (Figure 4A).

Eye movements directed toward the attention cue during intended fixation may confound behavioral measurements of spatial attention - for example, by increasing visual acuity for processing the target when it is presented at the cued location. Our behavioral results corroborate this concern (at least for paradigms similar to our spatial attention cuing experiment with a high cue validity and long cue-target SOA). Consistent with the fact that larger eye movements were primarily directed toward the attention cue prior to fixation training (the upper row in Figure 4), observers who made larger saccades produced stronger cuing effects, as was indicated by the significant positive correlation between the average amplitude of saccades and the cuing effect (cue-invalid RT minus cue-valid RT) $[r=$ $.49, t(34)=3.27, p<.003]$ (Figure 5A). Importantly, the informed-flicker training substantially reduced the average amplitude of saccades during intended fixation for all observers to within $\sim 1^{\circ}$ (the filled circles in Figure 5B) and eliminated systematic eye movements toward the attention cue (the lower panel in Figure 4A).

In summary, a few minutes of training using the informed-flicker condition dramatically improved central eye fixation, substantially reducing both the number (the left graph in Figure 3A) and amplitude (reducing to only $\sim 0.5^{\circ}$ on average; the left graph in Figure $3 \mathrm{~B}$ ) of undesirable eye movements throughout a relatively long $(3,200-$ $\mathrm{msec})$ period of intended central fixation. This training also eliminated systematic eye movements toward a peripheral attention cue (the lower panel in Figure 4A).

\section{DISCUSSION}

People constantly make saccadic eye movements (e.g., Yarbus, 1967) and are often unaware of making them. Our results demonstrate that providing real-time feedback about involuntary eye movements using a flicker display rapidly and dramatically improves people's ability to maintain central eye fixation, presumably through making them aware of sensations related to eye movements. The improvement is impressive because the informed-flicker training produced accurate central eye fixation in each observer (reducing his or her average saccade amplitudes 


\section{A Fixation Training or Control}

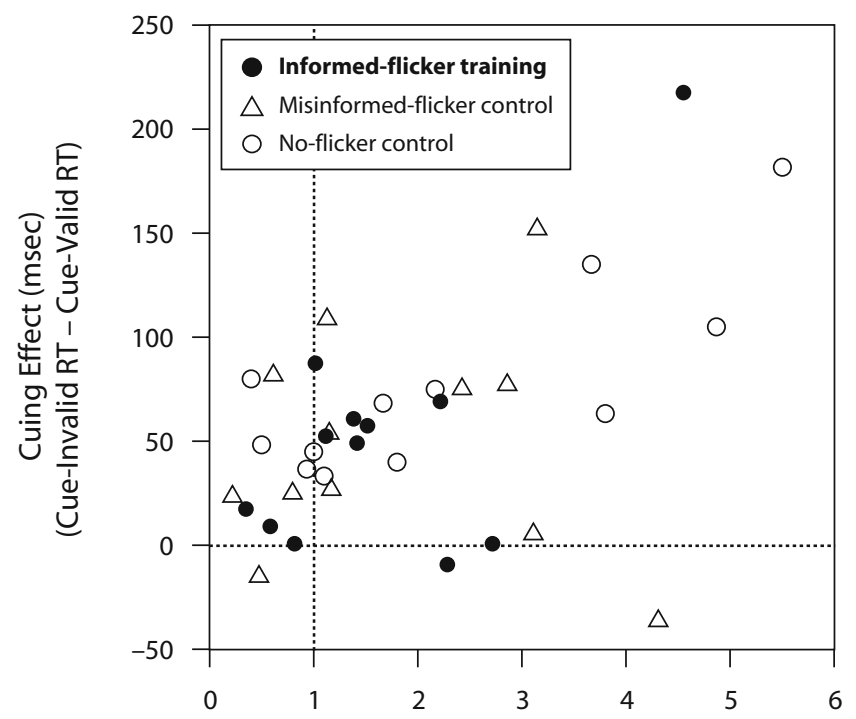

After

B Fixation Training or Control

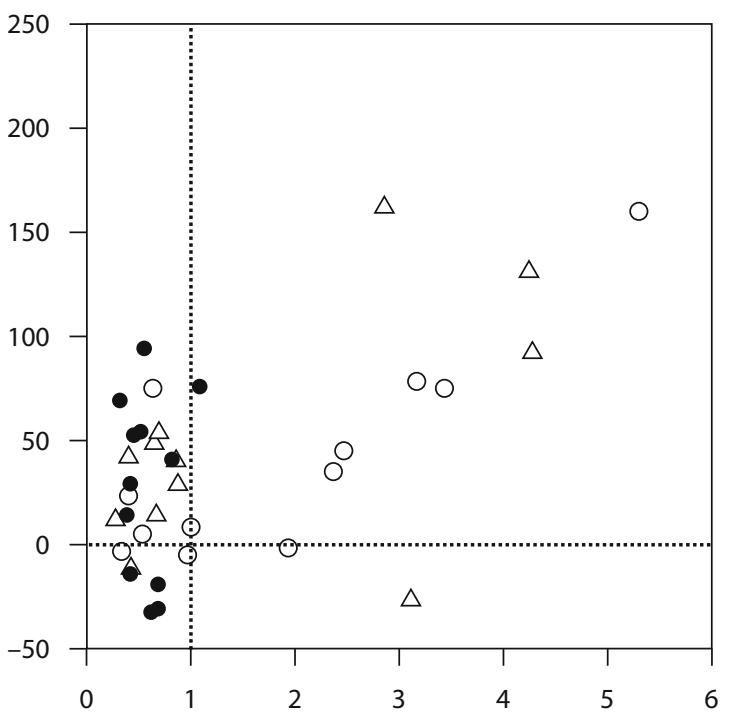

Average Amplitude of Saccades (deg)

Figure 5. Scatterplots showing the relationship between the average amplitude of saccades (in degrees of visual angle) and the cuing effect (cue-invalid response time [RT] minus cue-valid RT), before $(A)$ and after $(B)$ the fixation training or control. Different symbols represent the data from observers who underwent different conditions: The filled circles represent the informed-flicker training, the open triangles represent the misinformed-flicker control, and the open circles represent the no-flicker control. Note that the average saccade amplitudes for all observers who underwent the informed-flicker training (filled circles) reduced to $\sim 1^{\circ}$ after the training (see $B$ ).

to less than $\sim 1^{\circ}$ and eliminating systematic saccades toward the attention cue) in an attention task that was specially designed to make it difficult to maintain central eye fixation: We used an abrupt-onset peripheral cue with a high cue validity and a long SOA between the cue and the target. We recommend that a few minutes of this flickerbased fixation training be added prior to experiments that are sensitive to eye-movement artifacts.

\section{AUTHOR NOTE}

The first two authors made equal contributions to this project. This research was supported by National Institutes of Health Grant R01 EY018197 and National Science Foundation Grant BCS 0643191. Correspondence concerning this article should be sent to S. Suzuki, Department of Psychology, Northwestern University, 2029 Sheridan Rd., Evanston, IL 60208 (e-mail: satoru@northwestern.edu).

\section{REFERENCES}

Forte, J., Hogben, J. H., \& Ross, J. (1999). Spatial limitations of temporal segmentation. Vision Research, 39, 4052-4061. doi:10.1016/ S0042-6989(99)00124-8

Funes, M. J., LupiáñeZ, J., \& Milliken, B. (2005). The role of spatial attention and other processes on the magnitude and time course of cueing effects. Cognitive Processing, 6, 98-116. doi:10.1007/S10339 $-004-0038-7$

Hershberger, W. (1987). Saccadic eye movements and the perception of visual direction. Perception \& Psychophysics, 41, 35-44.

Steinman, R. M., Haddad, G. M., Skavenski, A. A., \& Wyman, D. (1973). Miniature eye movement. Science, 181, 810-819. doi:10.1126/ science. 181.4102 .810

Yarbus, A. L. (1967). Eye movements and vision. New York: Plenum.

(Manuscript received August 7, 2008; revision accepted for publication December 10, 2008.) 